\title{
Verleihung der Ehrenmitgliedschaft der Deutschen Röntgengesellschaft an Prim. Univ.-Prof. Dr. Walter Hruby
}

Auf dem Deutschen Röntgenkongress 2012 in Hamburg wurde Prof. Hruby in Anerkennung seiner Verdienste um die Informationstechnologie in der Radiologie mit der Ehrenmitgliedschaft der Deutschen Röntgengesellschaft ausgezeichnet. Prim. Univ.-Prof. Dr. Walter Hruby wurde nach Studium, Promotion und Facharztausbildung in Wien 1988 designierter Vorstand des Instituts für Röntgendiagnostik im Donauspital des Sozialmedizinischen Zentrums Ost der Stadt Wien. 1992 nahm das Institut seinen klinischen Betrieb auf, das Hruby bis heute leitet. Nach seiner Habilitation 1990 zum Universitätsprofessor folgte 1995 die Ernennung zum tit. A. o. Univ.-Professor der Medizinischen Universität Wien. 2008 wurde er mit dem goldenen Ehrenzeichen der Stadt Wien ausgezeichnet. Neben den vielen weiteren wissenschaftlichen und organisatorischen Tätigkeiten und Auszeichnungen ist Prof. Hruby Past President der Österreichischen Röntgengesellschaft und war 2011 Kongresspräsident des 92. Deutschen und 6. Gemeinsamen Röntgenkongresses der DRG und ÖRG.

Ein Hauptschwerpunkt seines Lebenswerkes ist die Implementierung der IT in der Radiologie: Als Vorreiter an der Schnittstelle zwischen Radiologie und der IT hat er sich im Fachgebiet einen bekannten Namen gemacht, und feiert darüber hinaus ein besonderes Jubiläum: Das Donauspital (SMZ Ost) ging genau vor 20 Jahren unter seiner Leitung als erste komplett digitalisierte Institution in Betrieb. Der Erfolg, den Walter Hruby bei der Entwicklung der IT-Technik für die Radiologie hatte, war wohl auch dadurch bedingt, dass er stets mit beiden Beinen voll im klinischen Leben stand, und es ihm immer um die unmittelbare Anwendbarkeit und
Praktikabilität von Prozessen ging. So hatte er etwa einen weiteren medizinischen Schwerpunkt in der interventionellen Radiologie und war Mitbegründer der CIRSE 1985 in Wien.

Seinem Kernthema war somit auch der Highlight-Vortrag am Deutschen Röntgenkongress gewidmet: In seinen Ausführungen zur „Leistungseffizienz durch IT in der Radiologie: Erwartungen - Fakten - Mythen - Missing Links“, ging Hruby nicht nur auf den faszinierenden Einzug der IT-Technologie in die Radiologie ein und der selbstverständlich gewordenen Entwicklung der RIS/PACS Systeme. Er zeigte ebenso auch die Gefahren und Risiken auf, die es im Lauf der weiteren Fortschritte zu bewältigen gibt. Insbesondere Schutzmaßnahmen für personenbezogene Daten, Identität und Privatsphäre müssten gewährleistet werden. Interoperabilität und Gewährleistung rechtlicher Rahmenbedingungen für die Weiterentwicklung sind jene Prozesse an denen, jenseits der rein technischen Gegebenheiten, noch zu arbeiten ist, so Hruby.

Die klinischen, organisatorischen und medizinischen Anwendungen werden bereits uneingeschränkt genutzt, und auch die ökonomischen Aspekte sind laut Hruby effizient und messbar geworden. Die gesellschaftlichen Auswirkungen sind aber noch nicht absehbar. Auch diese in einen für alle positiven Fluss zu leiten, ist sein beständiges Ziel.

Einiges bleibt für Hruby auch noch unerreicht: Zu den „missing links“ zählt für ihn die Vereinheitlichung der Plattformen und der Datenbanken. DICOM-Eigenschaften müssten im Detail abgestimmt und individuell geprüft werden. Ferner sollten Bedienungsoberflächen innerhalb der Trägerorganisationen vereinheitlicht werden - nicht nur in der Radiologie, sondern etwa auch auf der Intensivstation und im Katheterlabor ebenso wie in der Laborautomatisierung und Dokumentation. Die Informationen zum jeweiligen Patienten und die verfügbaren Programmfunktionen sollten abteilungsunabhängig dargestellt und nutzbar sein; Lösungen mit selbsterklärenden Oberflächen und automatischer Menüführung sind hier Vorbilder.

Verbesserungen erwartet Prof. Hruby vor allem durch die Vereinheitlichung verschiedener Parameter für alle Untersuchungen, wobei vor allem die großen Datenvolumina die entscheidenden Hürden darstellen. Eine Lösung der Aufgaben sieht Prof. Hruby nur in weiterer unaufhörlicher und gemeinsamer Arbeit mit Entwicklern und Systembetreuern, um die Vereinheitlichung und auch Vereinfachung des „Werkzeugs“ IT im Sinne einer verbesserten Patientenversorgung zu bewirken und sieht hierbei die Radiologie als Motor für die Anbieterseite.

Fortschritt wird nur dann stattfinden, so Hruby, wenn wir uns als Anwender kontinuierlich und konstruktiv einbringen.

Seinen ganzen Einsatz brachte Prof. Hruby in die Organisation des 92. Deutschen und 6. Gemeinsamen Röntgenkongresses der DRG und ÖRG 2011 ein. Als brillanter Diplomat in der radiologischen Nachbarschaftsbeziehung zwischen DRG und ÖRG präsentierte Prof. Hruby nach jahrelanger Vorarbeit eine von Freundschaft und Wissenschaft geprägte Präsidentschaft 2011. Ein Jahr danach erhielt er nun selbst die Anerkennung und Auszeichnung für sein Lebenswerk.

Für die ÖRG und das Präsidium herzlichst T. Rand und D. Tscholakoff 